Article

\title{
Existence and uniqueness of mild solution for stochastic partial differential equation with poisson jumps and delays
}

\author{
Annamalai Anguraj ${ }^{1, *}$ and Ravi Kumar ${ }^{1}$ \\ 1 Department of Mathematics, PSG College of Arts and Science, Coimbatore 641 046, India. \\ * Correspondence: angurajpsg@yahoo.com
}

Received: 13 May 2019; Accepted: 15 October 2019; Published: 16 November 2019.

\begin{abstract}
The objective of this paper is to investigate the existence and uniqueness theorem for stochastic partial differential equations with poisson jumps and delays. The existence of mild solutions of the problem is studied by using a different resolvent operator defined in [1] and fixed point theorem.
\end{abstract}

Keywords: Resolvent operator, mild solution, stochastic partial differential equations, poisson jumps, delays.

MSC: 34A12, 35R60, 47H10, 60H15.

\section{Introduction}

S tochastic differential equation is an emerging field drawing attention from both theoretical and applied disciplines, which has been successfully applied to problems in mechanical, electrical, physics, economics and several fields in engineering. For details see [2,3] and the references therein. Recently a large number of interesting results of stochastic equations have been reported in [4-8]. Stochastic differential equations are used in the modeling of real life phenomena, where there is a need for an aspect of randomness (see [9-11]).

Furthermore, several practical systems (such as sudden price variations due to market crashes, earthquakes, hurricanes, epidemics, and so on) experiences some jump type stochastic perturbations. The sample paths are not being continuous, thus it is seize considering stochastic processes with jumps in describing the models. Generally, the jump models are derived from poisson random measure. The sample paths of systems being right continuous possess left limits. In the recent trend, researchers are focusing more on the theory and applications of impulsive stochastic functional differential equations with poisson jumps. Precisely, existence and stability results on impulsive stochastic functional differential equations with poisson jumps are found in [12-15] and the references therein. Successively, few works have been reported in the study of stochastic differential equations with poisson jumps, refer to $[13,14,16]$.

However, motivated by the above consideration, the aim of this paper is to establish the results on existence and uniqueness for stochastic differential equation with Poisson jumps and delay of the form:

$$
\begin{aligned}
d u(t) & =[A u(t)+f(t, u(t-\rho(t)))] d t+g(t, u(t-\delta(t))) d W(t)+\int_{Z} h(t, u(t-\sigma(t)), z) \tilde{N}(d t, d z), \\
x_{0} & =\xi \in D_{\mathcal{F}_{0}}^{b}([-\tau, o], H) .
\end{aligned}
$$

The mappings $f: \mathbb{R}_{+} \times D([-\tau, o] ; H) \rightarrow H, g: \mathbb{R}_{+} \times D([-\tau, o] ; H) \rightarrow \mathbb{L}_{2}^{0}(K, H), h: \mathbb{R}_{+} \times D([-\tau, o] ; H) \times$ $Z \rightarrow H$ are Borel measurable, $\rho: \mathbb{R}_{+} \rightarrow[0, \tau], \delta: \mathbb{R}_{+} \rightarrow[0, \delta], \sigma: \mathbb{R}_{+} \rightarrow[0, \sigma]$ are continuous.

This paper is organized as follows: In Section 2, we give some basic definitions and results, which will be used in the sequel. In Section 3, the existence result for the system 1 is proved.

\section{Preliminaries}

Let $\mathbb{H}$ and $\mathbb{K}$ be a two real separable Hilbert space. Let $\mathcal{L}(\mathbb{H}, \mathbb{K})$ denote the space of all bounded linear operators from $\mathbb{H}$ into $\mathbb{K}$, equipped with the usual operator norm $\|$.$\| and we abbreviate this notation to \mathcal{L}(\mathbb{H})$ when $\mathbb{H}=\mathbb{K}$. In this paper, we always use the same symbol $\|$.$\| to denote norms of operators regardless of the$ spaces potentially involved when no confusion possibly arises. Let $\left(\Omega, \mathcal{F}, \mathcal{F}_{t \geq 0}, \mathbb{P}\right)$ be a complete probability 
space with a normal filtration $\mathcal{F}_{t \geq 0}$ satisfying the usual conditions (i.e. it is increasing and right continuous while $\mathcal{F}_{0}$ contains all $\mathbb{P}$ null sets).

Let $\{W(t): t \geq 0\}$ denote a $\mathbb{K}$-valued Wiener process defined on the probability space $\left(\Omega, \mathcal{F}, \mathcal{F}_{t \geq 0}, \mathbb{P}\right)$, independent of poisson point process with covariance operator $Q$; that is, $E\langle W(t), x\rangle_{\mathbb{K}}\langle W(s), y\rangle_{\mathbb{K}}=(t \wedge$ s) $\langle Q x, y\rangle_{\mathbb{K}}$, for all $x, y \in \mathbb{K}$, where $Q$ is a positive, self-adjoint, trace class operator on $\mathbb{K}$. In particular, we denote $W(t)$ a $\mathbb{K}$-valued $Q$-Wiener process with respect to $\mathcal{F}_{t \geq 0}$. To define stochastic integrals with respect to the $Q$-Wiener process $W(t)$, we introduce the subspace $\mathbb{K}_{0}=Q^{\frac{1}{2}} \mathbb{K}$ of $\mathbb{K}$ endowed with the inner product:

$$
\langle u, v\rangle_{\mathbb{K}_{0}}=\left\langle Q^{\frac{-1}{2}} u, Q^{\frac{-1}{2}} v\right\rangle_{\mathbb{K}}
$$

as a Hilbert space. We assumed that there exists a complete orthonormal system $e_{i}$ in $\mathbb{K}$, a bounded sequence of positive real numbers $\lambda_{i}$ such that $Q e_{i}=\lambda_{i} e_{i}, i=1,2,3, \ldots$ and a sequence $\beta_{i}(t)_{i>1}$ of independent standard Brownian motions such that $W(t)=\sum_{i=1}^{+\infty} \sqrt{\lambda_{i}} \beta_{i}(t) e_{i}$ for $t \geq 0$ and $\mathcal{F}_{t}=\mathcal{F}_{t}^{w}$, where $\mathcal{F}_{t}^{w}$ is the $\sigma$-algebra generated by $W(s): 0 \leq s \leq t$.

Suppose $\{p(t), t \geq 0\}$ is a $\sigma$-finite stationary $\mathcal{F}_{t}$ adapted Poisson point process taking values in measurable space $(U, \mathcal{B} U)$. The random measure $N_{p}$ defind by $N_{p}((o, t] \times \wedge):=\sum_{s \in(0, t]} I_{\wedge}(P(s))$ for $\wedge \in \mathcal{B}(U)$ is called the Poisson random measure induced by $P($.$) , thus, we can define the measure N$ by $N(d t, d y)=$ $N_{p}(d t, d y)-v(d y) d t$, where $y$ is the characteristic measure of $N_{p}$, which is called the compensated Poisson random measure. For a main source for the material on Poisson process and random measure we refer to [17]. For a Borel set $Z \in B_{\sigma}(\mathbb{H}-\{0\})$, we denote by $\mathcal{P}^{2}([0, T] \times Z ; \mathbb{H})$ the space of all predicable mapping $H:[0, T] \times Z \times \Omega \rightarrow \mathbb{H}$ for which $\int_{0}^{t} \int_{Z} E\|H(t, v)\|^{2} d t \lambda(d z)<\infty$. Then one can define the $\mathbb{H}$ - valued stochastic integral

$$
\int_{0}^{t} \int_{Z} H(t, v) \bar{N}(d t, d z)
$$

which is a centered square integrable martingale [18]. We always assume that $W(t)$ and $\bar{N}$ are independent of $\mathcal{F}_{0}$. Also $S=C([0, a] ; X)$ denotes the space of all continuous functions with the norm $\|\cdot\|_{C([0, a] ; X)}=$ $\sup _{t \in[0, a]}\|x(t)\|_{X}$.

Consider the following Stochastic partial differential equation driven by Poisson jumps with delays:

$$
d u(t)=[A u(t)+f(t, u(t-\rho(t)))] d t+g(t, u(t-\delta(t))) d W(t)+\int_{Z} h(t, u(t-\sigma(t)), z) \tilde{N}(d t, d z) .
$$

The above equation is equivalent to the following integral equation:

$$
\begin{aligned}
u(t)= & \xi(0)+\int_{0}^{t} A u(t) d s+\int_{0}^{t} f(s, u(s-\rho(s))) d s+\int_{0}^{t} g(s, u(s-\delta(s))) d W(s) \\
& +\int_{0}^{t} \int_{Z} h(s, u(s-\sigma(s)), z) \tilde{N}(d s, d z)
\end{aligned}
$$

This can be written the following form:

$$
u(t)=f(t)+\int_{0}^{t} s^{\prime}(t-s) f(s) d s
$$

where,

$$
\begin{aligned}
f(t)= & \xi(0)+\int_{0}^{t} f(s, u(s-\rho(s))) d s+\int_{0}^{t} g(s, u(s-\delta(s))) d W(s) \\
& +\int_{0}^{t} \int_{Z} h(s, u(s-\sigma(s)), z) \tilde{N}(d s, d z) .
\end{aligned}
$$

Let us assume that the integral equation (3) has an associated resolvent operator $\{S(t)\}_{t \geq 0}$ on $H$.

Definition 1. [1] A family $(S(t))_{t \geq 0} \subset \mathcal{L}(X)$ of bounded linear operators in $X$ is called resolvent for (4)(or solution operator for (4), if the following conditions are satisfied

(S1) $S(t)$ is strongly continuous on $\mathbb{R}^{+}$and $S(0)=I$, 
(S2) $S(t)$ commutes with $A$, which means that $S(t) \mathcal{D}(A) \subset \mathcal{D}(A)$ and $A S(t) x=S(t) A x$ for all $x \in \mathcal{D}(A)$ and $t \geq 0$;

(S3) The resolvent equation holds

$$
S(t) x=x+\int_{0}^{t} A s(s) x d s
$$

Definition 2. [1] A resolvent $S(t)$ for (3) is called differentiable, if $S(\cdot) x \in W^{1,1}\left(\mathbb{R}^{+} ; X\right)$ for each $x \in \mathcal{D}(A)$ and there is $\phi_{A} \in L_{\text {loc }}^{1}\left(\mathbb{R}^{+}\right)$such that $\left\|S^{\prime}(t) x\right\| \leq \phi_{A}(t)\|x\|_{\mathcal{D}(A)}$ a.e. on $\mathbb{R}^{+}$, for each $x \in \mathcal{D}(A)$, Where the notation $[\mathrm{D}(\mathrm{A})]$ stands the domain of the operator $A$ provided with the graph norm $\|x\|_{[D(A)]}=\|x\|+\|A x\|$.

Lemma 3. [1] Suppose (3) admits a differentiable resolvent $S(t)$ and if $f \in C([0, a] ; \mathcal{D}(A))$, then

$$
u(t)=f(t)+\int_{0}^{t} S^{\prime}(t-s) f(s) d s, t \in[0, a]
$$

is a mild solution of (3).

In order to prove that the existence result of the stochastic partial differential equation with poisson jumps and delays, we need the following assumptions:

(H1) The mapping $f(t,$.$) and g(,$.$) satisfies the following Lipschitz and linear growth conditions, for any$ $x, y \in \mathbb{H}$ and $t \geq 0$

$$
\begin{array}{r}
\|f(t, x)-f(t, y)\|_{\mathbb{H}} \leq L_{1}\|x-y\|_{\mathbb{H}} \forall t \geq 0, x, y \in \mathbb{H} \text { where } L_{1}>0 \\
\|g(t, x)-g(t, y)\|_{\mathbb{H}} \leq L_{1}\|x-y\|_{\mathbb{H}} \forall t \geq 0, x, y \in \mathbb{H} \text { where } L_{2}>0 .
\end{array}
$$

(H2) The mapping $h(t,$.$) satisfy global Lipschitz conditions, for any x, y \in \mathbb{H}$ and $t \geq 0$

$$
\int_{Z}\|h(t, x, z)-h(t, y, z)\|^{2} v(d z) \leq L_{3}^{2}\|x-y\|^{2}, L_{3}>0 .
$$

\section{Existence and uniqueness results}

In this section, we provide the existence results for (1), this problem is equivalent to the following integral equation:

$$
\begin{aligned}
u(t)= & \xi(0)+\int_{0}^{t} A u(t) d s+\int_{0}^{t} f(s, u(s-\rho(s))) d s+\int_{0}^{t} g(s, u(s-\delta(s))) d W(s) \\
& +\int_{0}^{t} \int_{Z} h(s, u(s-\sigma(s)), z) \tilde{N}(d s, d z) .
\end{aligned}
$$

By Lemma 2 and the above representation, the mild solution of (1) can be defined as follows:

Definition 4. A stochastic process $\{u(t), t \in[0, T]\}, 0 \leq T \leq \infty$, is called a mild solution of (1) if

1. $u(t)$ is adapted to $\mathcal{F}_{t}, t \geq 0$;

2. $u(t) \in \mathbb{H}$ has cadlag paths on $t \in[0, T]$ almost surely, and for arbitrary $0 \leq t \leq T$,

$$
\begin{aligned}
u(t)= & \xi(0)+\int_{0}^{t} f[s, u(s-\rho(s))] d s+\int_{0}^{t} g[s, u(s-\delta(s))] d W(s) \\
& +\int_{0}^{t} \int_{Z} h[s, u(s-\sigma(s)), z] \tilde{N}(d s d z)+\int_{0}^{t} s^{\prime}(t-s) \xi(0) d s+\int_{0}^{t} s^{\prime}(t-s) \int_{0}^{s} f[\tau, x(\tau-\rho(\tau))] d \tau d s \\
& +\int_{0}^{t} s^{\prime}(t-s) \int_{0}^{s} g[\tau, u(\tau-\delta(\tau))] d W(\tau) d s+\int_{0}^{t} s^{\prime}(t-s) \int_{0}^{s} \int_{Z} h[\tau, u(\tau-\sigma(\tau)), z] \tilde{N}(d s d z) d s .
\end{aligned}
$$

Theorem 5. Assume that (H1) and (H2)are hold, then the problem (1) is a unique mild solution. 
Proof. Define the operator $\Phi: S \rightarrow S$ by $\Phi(u)(t)=\xi(t)$ for $t \in[-\tau, 0]$ and for $t \geq 0$, defined by

$$
\begin{aligned}
\Phi(u)(t)= & \xi(0)+\int_{0}^{t} f[s, u(s-\rho(s))] d s+\int_{0}^{t} g[s, u(s-\delta(s))] d W(s) \\
& +\int_{0}^{t} \int_{Z} h[s, u(s-\sigma(s)), z] \tilde{N}(d s d z)+\int_{0}^{t} s^{\prime}(t-s) \xi(0) d s \\
& +\int_{0}^{t} s^{\prime}(t-s) \int_{0}^{s} f[\tau, x(\tau-\rho(\tau))] d \tau d s+\int_{0}^{t} s^{\prime}(t-s) \int_{0}^{s} g[\tau, u(\tau-\delta(\tau))] d w(\tau) d s \\
& +\int_{0}^{t} s^{\prime}(t-s) \int_{0}^{s} \int_{Z} h[\tau, u(\tau-\sigma(\tau)), z] \tilde{N}(d s d z) d s .
\end{aligned}
$$

First, we verify that $\Phi$ is $\mathrm{p}$-th mean continuous on $[0, \infty)$. Let $u \in S, t_{1} \geq 0$ and $|h|$ be sufficiently small, then

$$
E\left\|(\Phi u)\left(t_{1}+h\right)-(\Phi u)\left(t_{1}\right)\right\|_{H}^{P} \leq 7^{p-1} \sum_{i=1}^{7} E\left\|I_{i}\left(t_{1}+h\right)-I_{i}\left(t_{1}\right)\right\|_{H}^{P}
$$

By using Hölders inequalities and the Burkholder-Davies-Gundy inequality, we have

$$
\begin{aligned}
E\left\|I_{2}\left(t_{1}+h\right)-I_{2}\left(t_{1}\right)\right\|_{H}^{P} & \leq E\left\|\int_{0}^{t_{1}+h} g[s, u(s-\delta(s))] d W(s)-\int_{0}^{t_{1}} g[s, u(s-\delta(s))] d W(s)\right\|_{H}^{P} \\
& \leq E\left\|\int_{t_{1}}^{t_{1}+h} g[s, u(s-\delta(s))] d w(s)\right\|_{H}^{P} \\
& \leq c_{p} E\left(\left(\int_{t_{1}}^{t_{1}+h}\|g[s, u(s-\delta(s))]\|_{H}^{P}\right)^{2 / p} d s\right)^{p / 2} \\
& \leq c_{p}\left(\int_{t_{1}}^{t_{1}+h} E\|g[s, u(s-\delta(s))]\|_{H}^{P} d s\right) \rightarrow 0 \text { as } h \rightarrow 0 .
\end{aligned}
$$

Next

$$
\begin{aligned}
E\left\|I_{7}\left(t_{1}+h\right)-I_{7}\left(t_{1}\right)\right\|_{H}^{P} \leq & 2^{p-1} E\left\|\int_{0}^{t_{1}} s^{\prime}\left(t_{1}+h-s\right)-s^{\prime}\left(t_{1}-s\right)\left[\int_{0}^{s} \int_{Z} h[\tau, u(\tau-\sigma(\tau)), z] \bar{N}(d \tau d z)\right] d s\right\|_{H}^{P} \\
& +2^{p-1} E\left\|\int_{t_{1}}^{t_{1}+h} s^{\prime}\left(t_{1}+h-s\right) \int_{0}^{s} \int_{Z} h[\tau, u(\tau-\sigma(\tau)), z] \bar{N}(d \tau d z) d s\right\|_{H}^{P} \\
& \rightarrow 0 \text { as } h \rightarrow 0 .
\end{aligned}
$$

Similarly, we can verify that:

$$
E\left\|I_{i}\left(t_{1}+h\right)-I_{i}\left(t_{1}\right)\right\|_{H}^{2} \rightarrow 0, \quad i=1,3,4,5,6 \text { as } h \rightarrow 0,
$$

where $c_{p}=(p(p-1) / 2)^{p / 2}$. Thus $\Phi$ is indeed continuous in pth mean on $[0, \infty)$.

Next, we show that $\Phi(S) \subset S$. It follows from (1), then we have

$$
\begin{aligned}
E\|(\Phi x)(t)\|_{H}^{P} \leq & 8^{P-1} E\|\xi(0)\|_{H}^{P}+8^{P-1} E\left\|\int_{0}^{t} f[s, u(s-\rho(s))] d s\right\| \\
& +8^{P-1} E\left\|\int_{0}^{t} g[s, u(s-\delta(s))] d W(S)\right\|_{H}^{P}+8^{P-1} E\left\|\int_{0}^{t} \int_{Z} h[s, u(s-\sigma(s)), z] \bar{N}(d s d z)\right\|_{H}^{P} \\
& +8^{P-1} E\left\|\int_{0}^{t} s^{\prime}(t-s) \xi(0) d s\right\|_{H}^{P}+8^{P-1} E\left\|\int_{0}^{t} s^{\prime}(t-s) \int_{0}^{s} f[\tau, u(\tau-\rho(\tau))] d \tau d s\right\|_{H}^{P} \\
& +8^{P-1} E\left\|\int_{0}^{t} s^{\prime}(t-s) \int_{0}^{s} g[\tau, u(\tau-\delta(\tau))] d W(\tau) d s\right\|_{H}^{P} \\
& +8^{P-1} E\left\|\int_{0}^{t} s^{\prime}(t-s) \int_{0}^{s} \int_{Z} h[\tau, u(\tau-\sigma(\tau)), z] \bar{N}(d \tau d z) d s\right\|_{H}^{P}=\sum_{i=1}^{8} J_{i}(t) .
\end{aligned}
$$


Now we estimate $J_{i}, i=1,2, \ldots, 8$, then we have

$$
J_{1}(t) \leq\|\xi\|_{D}^{P}<\infty
$$

Now by (H1), we obtain

$$
J_{2}(t) \leq E\left[\int_{0}^{t}\|f[s, u(s-\rho(s))]\|_{H} d s\right]^{P} \leq L_{1}^{p}\|u\|_{D}^{p} T .
$$

Now, from the Lemma (Da Prato and Zabczyk [4]) and by the (H1), we have

$$
J_{3}(t) \leq c_{p}\left[\int_{0}^{t}\left(E\|g[s, u(s-\delta(s))]\|_{H}^{P}\right)^{\frac{2}{p}} d s\right]^{\frac{p}{2}} \leq c_{p} L_{2}^{P}\|u\|_{D}^{P} T .
$$

Similarly, by (H2), we obtain

$J_{4}(t) \leq c_{p}\left[E\left[\int_{0}^{t} \int_{h} \| h(s, u(s-\sigma(s))), z\right] \|_{H}^{2} d s v(d z)\right]^{\frac{p}{2}} \leq c_{p} L_{3}^{p}\left[\int_{0}^{t} E\|u(s-\sigma(s))\|_{H}^{2} d s\right]^{\frac{p}{2}} \leq c_{p} L_{3}^{p}\|u\|_{D}^{P} T$

By (H1), (H2) and Well known Lemma (Da Prato and Zabczyk [4]), we have

$$
\begin{gathered}
J_{5}(t) \leq\|\xi(0)\|\left\|\phi_{A}\right\|_{L^{1}\left([0, t] ; \mathbb{R}^{+}\right)} \\
J_{6}(t) \leq L_{1}^{P} \int_{0}^{t} \phi_{A}(t-s) \int_{0}^{t} E\|u(\tau-\rho(\tau)) d \tau\|_{H}^{p} d s \leq\|\xi(0)\|\left\|\phi_{A}\right\|_{L^{1}\left([0, t] ; \mathbb{R}^{+}\right)} \\
J_{7}(t) \leq c_{p} L_{2}^{p} \int_{0}^{t} \phi_{A}(t-s) \int_{0}^{s} E\|u(\tau-\delta(\tau))\|_{H}^{p} d \tau d s \leq c_{p} L_{2}^{p}\|u\|_{D}^{p} T\left\|\phi_{A}\right\|_{L^{1}\left([0, t] ; \mathbb{R}^{+}\right)} \\
J_{8}(t) \leq c_{p} E\left[\int_{0}^{t} \phi_{A}(t-s) \int_{0}^{s} \int_{Z}\|h[\tau, u(\tau-\sigma(\tau)), z]\|^{2} v(d z) d s\right]^{\frac{p}{2}} \leq c_{p} L_{3}^{p}\|u\|_{D}^{p} T\left\|\phi_{A}\right\|_{L^{1}\left([0, t] ; \mathbb{R}^{+}\right)}
\end{gathered}
$$

From the above estimations, we have $\|(\Phi x)(t)\|<\infty$. So we conclude that $\Phi(S) \subset(S)$.

Next, we need to show that $\Phi$ is contraction mapping. Let $u, v \in S$, then we have

$$
\begin{aligned}
& E \sup _{t \in[0, T]}\|(\Phi u)(t)-(\Phi v)(t)\| \\
& \leq 6^{p-1} \sup _{t \in[0, T]} E\left\|\int_{0}^{t}(f[s, u(s-\rho(s))]-f[s, v(s-\rho(s))]) d s\right\|_{H}^{P} \\
& +6^{p-1} \sup _{t \in[0, T]} E\left\|\int_{0}^{t}(g[s, u(s-\delta(s))]-g[s, v(s-\delta(s))]) d W(s)\right\|_{H}^{P} \\
& +6^{p-1} \sup _{t \in[0, T]} E\left\|\int_{0}^{t} \int_{Z}(h[s, u(s-\sigma(s)), z]-h[s, v(s-\sigma(s)), z]) \tilde{N}(d s, d z)\right\|_{H}^{P} \\
& +6^{p-1} \sup _{t \in[0, T]} E\left\|\int_{0}^{t} s^{\prime}(t-s)\left[\int_{0}^{s}(f[\tau, u(\tau-\rho(\tau))]-f[\tau, v(\tau-\rho(\tau))]) d \tau\right] d s\right\|_{H}^{P} \\
& +6^{p-1} \sup _{t \in[0, T]} E\left\|\int_{0}^{t} s^{\prime}(t-s)\left[\int_{0}^{s}(g[\tau, u(\tau-\delta(\tau))]-g[\tau, v(\tau-\delta(\tau))]) d W(\tau)\right] d s\right\|_{H}^{P} \\
& +6^{p-1} \sup _{t \in[0, T]} E\left\|\int_{0}^{t} s^{\prime}(t-s)\left[\int_{0}^{s} \int_{z}(h[\tau, u(\tau-\sigma(\tau)), z]-h[\tau, v(\tau-\sigma(\tau)), z]) \tilde{N}(d \tau d z)\right] d s\right\|_{H}^{P} \\
& \leq 6^{p-1} \sup _{t \in[0, T]} E\|u(t)-v(t)\|_{H}^{P} T \times\left(L_{1}^{P}++c_{p} L_{2}^{P}+L_{3}^{P}\right)\left[1+\left\|\phi_{A}\right\|_{L^{1}[0, T] ; \mathbb{R}^{+}}\right] .
\end{aligned}
$$


If $T>0$ is sufficiently small, then we can ensure that

$$
\left(L_{1}^{P}++c_{p} L_{2}^{P}+L_{3}^{P}\right)\left[1+\left\|\phi_{A}\right\|_{L^{1}[0, T] ; \mathbb{R}^{+}}\right]<1 .
$$

We conclude that the operator $\Phi$ satisfies the contraction mapping principle and hence there exists a unique mild solution of (1) on $T \in[0, T]$.

Author Contributions: All authors contributed equally to the writing of this paper. All authors read and approved the final manuscript.

Conflicts of Interest: "The authors declare no conflict of interest."

\section{References}

[1] Präss, J. (2013). Evolutionary integral equations and applications (Vol. 87). Birkhäuser Verlag, Basel.

[2] Karatzas,I., \& Shreve, S. E. (1991). Brownian Motion and Stochastic calculus. Springer- Verlag, Berlin.

[3] Mao, X. (2007). Stochastic differential equations and applications. Ellis Horwood, Chichester, UK.

[4] Da Prato, G., \& Zabczyk, J. (2014). Stochastic equations in infinite dimensions. Cambridge university press.

[5] Hale, J. K., \& Lunel, S. M. V. (2013). Introduction to functional differential equations (Vol. 99). Springer Science \& Business Media.

[6] Taniguchi, T., Liu, K., \& Truman, A. (2002). Existence, uniqueness, and asymptotic behavior of mild solutions to stochastic functional differential equations in Hilbert spaces. Journal of Differential Equations, 181(1), 72-91.

[7] Xu, D., Yang, Z., \& Huang, Y. (2008). ExistenceÜuniqueness and continuation theorems for stochastic functional differential equations. Journal of Differential Equations, 245(6), 1681-1703.

[8] Ren, Y., \& Chen, L. (2009). A note on the neutral stochastic functional differential equation with infinite delay and Poisson jumps in an abstract space. Journal of Mathematical Physics, 50(8), 082704.

[9] Balasubramaniam, P., \& Ntouyas, S. K. (2006). Controllability for neutral stochastic functional differential inclusions with infinite delay in abstract space. Journal of Mathematical Analysis and Applications, 324(1), 161-176.

[10] Mokkedem, F. Z., \& Fu, X. (2017). Approximate Controllability for a Semilinear Stochastic Evolution System with Infinite Delay in $L_{p}$ Space. Applied Mathematics \& Optimization, 75(2), 253-283.

[11] Shukla, A., Arora, U., \& Sukavanam, N. (2015). Approximate controllability of retarded semilinear stochastic system with non local conditions. Journal of Applied Mathematics and Computing, 49(1-2), 513-527.

[12] Cui, J., Yan, L., \& Sun, X. (2011). Exponential stability for neutral stochastic partial differential equations with delays and Poisson jumps. Statistics \& Probability Letters, 81(12), 1970-1977.

[13] Annamalai, A., Kandasamy, B., Baleanu, D., \& Arumugam, V. (2018). On neutral impulsive stochastic differential equations with Poisson jumps. Advances in Difference Equations, 2018(1), 290.

[14] Boufoussi, B., \& Hajji, S. (2010). Successive approximation of neutral functional stochastic differential equations with jumps. Statistics \& probability letters, 80(5-6), 324-332.

[15] Cui, J., \& Yan, L. (2012). Successive approximation of neutral stochastic evolution equations with infinite delay and Poisson jumps. Applied Mathematics and Computation, 218(12), 6776-6784.

[16] Diop, M. A., \& Zene, M. M. (2016). On the asymptotic stability of impulsive neutral stochastic partial integrodifferential equations with variable delays and Poisson jumps. Afrika Matematika, 27(1-2), 215-228.

[17] J.C.F. Kingman (1993). Poisson Processes. Oxford University Press.

[18] Luo, J., \& Liu, K. (2008). Stability of infinite dimensional stochastic evolution equations with memory and Markovian jumps. Stochastic Processes and their Applications, 118(5), 864-895.

(C) 2019 by the authors; licensee PSRP, Lahore, Pakistan. This article is an open access article distributed under the terms and conditions of the Creative Commons Attribution (CC-BY) license (http://creativecommons.org/licenses/by/4.0/). 